\title{
How Our Editorial Process Works
}

The objective of this editorial is to remind ourselves how our journal's editorial process works. We should remember that in the past year quite a few changes have taken place and some of them affect how this process is implemented.

Our journal, the Annals of Biomedical Engineering, is an interdisciplinary, international journal which presents original and review articles in the major fields of bioengineering and biomedical engineering. It is the flagship journal of the Biomedical Engineering Society and all of us are working diligently to turn it into the flagship journal of our entire field.

A major aim of bioengineering in general is to identify or develop integrated approaches to provide solutions to vexing biological and biomedical problems. The philosophy is to employ engineering approaches that enhance the power of the scientific method, and to maintain a balance between experimental observation and quantitative analyses.

While the development of theory and of mathematical models is strongly endorsed, these should be evaluated wherever possible using biological data from experiments that test specific hypotheses. Thus, please keep in mind that $A B M E$ discourages submissions of routine computational simulations that produce easily anticipated results, lack experimental validation, or represent incremental advancements of understanding.

Papers must be submitted via upload in a word processing format, preferably in Microsoft Word. Authors can also submit LaTex manuscripts through our online submission process. Our software can accept LaTeX manuscripts, and usually formats them properly when converting them to PDF. However, Microsoft Word files are preferred for upload.

After an author has submitted a manuscript and approved the PDF, Springer staff check to make sure it is complete. Manuscripts are also checked for plagiarism using CrossCheck. CrossCheck is a webbased tool that can identify matching text and allow one to determine if plagiarism has occurred.

Next, the manuscript is assigned to the Editorin-Chief, who checks to make sure the manuscript adheres to the aims and scope of the Annals, and to our manuscript guidelines. Manuscripts that fall outside ABME's scope will be rejected without review. As a general interest journal, some otherwise outstanding articles may be rejected because we deem them more suitable for a specialized journal. We may suggest authors submit some of these articles to BMES's more specialized publications, Cardiovascular Engineering and Technology or Cellular and Molecular Bioengineering.

We also reject without review manuscripts that fail to adhere to our guidelines. The full guidelines are posted on ABME's website. Manuscripts must not exceed approximately 20 double-spaced pages, including references but not figures or tables. Each manuscript must be uploaded in a single file. The manuscript must have 1" margins and 12 point Times New Roman or Arial font. Though the page count includes references but not figures or tables, authors should limit figures to a manageable number (6-8 should suffice). All text must be double-spaced, including footnotes, references, legends, and tables. Tables and figures should be referred to in the manuscript in a sequential manner. Please note that we rarely make exceptions to these guidelines.

Manuscripts that pass this first round of scrutiny are assigned by the Editor-in-Chief to an Associate Editor, who will handle the review. We invite Associate Editors whose expertise most closely matches the manuscript but please note that not all Associate Editors will be experts for all manuscripts they receive. The Associate Editor invites reviewers, and makes sure that it receives three reviews. The Managing Editor works closely with the Associate Editors and Reviewers to make sure the process runs smoothly and 
efficiently. So far in 2010, Reviewers have been completing reviews in an average of 14 days.

When all reviews are complete, the Associate Editor makes a recommendation to the Editorin-Chief, who makes the final recommendation. On average, manuscripts complete the editorial process in about 20 days.

As indicated in last month's editorial, we are making significant strides but our collective work will be incomplete unless our impact in the field is significant (as measured by the appropriate impact factor indices) and sustained. We are all volunteers in this quest but we do it because we love our scientific field and because we are passionate about excellence. Thank you.

Respectfully submitted, K. A. Athanasiou

Davis, CA

\section{OPEN ACCESS}

This article is distributed under the terms of the Creative Commons Attribution Noncommercial License which permits any noncommercial use, distribution, and reproduction in any medium, provided the original author(s) and source are credited. 